VoL. 47 (1993) [321-332]

\title{
ON UNION-CLOSED SETS AND CONWAY'S SEQUENCE
}

\section{J-C. Renaud and L.F. Fitina}

In 1991 Renaud defined a boundary function $\phi(n)$ for union-closed sets, and evaluated it to $n=17$. Also in 1991, Mallows examined a sequence $a(n)$ defined recursively by Conway in 1988 .

Investigation of some properties of strictly reduced ordered power sets, a class of union-closed sets, leads to the conclusion that $a(n+1)$ is an upper bound for $\phi(n)$, and the union-closed sets conjecture holds if the conjecture $\phi(n)=a(n+1)$ is valid.

\section{INTRODUCTION}

For the purposes of this paper a union-closed set, henceforth called a collection, is defined as a non-empty finite collection of non-empty finite sets, closed under union. It has been conjectured that in any union-closed collection there exists at least one element which occurs in more than half the sets. (This was first proposed, in slightly different form, in 1979 by Frankl [3, p.525]: in the original conjecture the null set is assumed to be in the collection, and it is conjectured that there exists an element in at least half the sets.)

While investigating this conjecture, Renaud in [2] defined the boundary function $\phi(n)$ on positive integers by $\phi(n)=k$ if all union-closed collections of $n$ sets have an element in $k$ sets and there exists a union-closed collection of $n$ sets in which no element occurs in $k+1$ sets. The first 17 values of $\phi(n)$ were evaluated:

\begin{tabular}{|c|ccccccccccccccccc|}
\hline$n$ & 1 & 2 & 3 & 4 & 5 & 6 & 7 & 8 & 9 & 10 & 11 & 12 & 13 & 14 & 15 & 16 & 17 \\
$\phi(n)$ & 1 & 2 & 2 & 3 & 4 & 4 & 4 & 5 & 6 & 7 & 7 & 8 & 8 & 8 & 8 & 9 & 10 \\
\hline
\end{tabular}

Coincidentally, in [1] Mallows investigated a sequence $a(n)$ defined in 1988 by Conway: $a(1)=1, a(2)=1$, and for $n>2 a(n)=a(a(n-1))+a(n-a(n-1))$. The matching first values of $a(n)$ are

\begin{tabular}{|c|cccccccccccccccccc|}
\hline$n$ & 1 & 2 & 3 & 4 & 5 & 6 & 7 & 8 & 9 & 10 & 11 & 12 & 13 & 14 & 15 & 16 & 17 & 18 \\
$a(n)$ & 1 & 1 & 2 & 2 & 3 & 4 & 4 & 4 & 5 & 6 & 7 & 7 & 8 & 8 & 8 & 8 & 9 & 10 \\
\hline
\end{tabular}

Received 27 April 1992

Copyright Clearance Centre, Inc. Serial-fee code: 0004-9729/93 \$A2.00+0.00. 
It is immediately conjecturable that $\phi(n)=a(n+1)$. If this could be proven then the validity of the union-closed sets conjecture would follow, since Mallows shows that $a(n) \geqslant n / 2$ for all $n \geqslant 1$. Unfortunately this has not yet been achieved - in this paper only the partial result $\phi(n) \leqslant a(n+1)$ is obtained. However, it leads to another avenue: strictly reduced ordered power sets are defined, and it is shown that these are union-closed collections of a certain type, with the element occuring most frequently in such collections of $n$ sets occuring in exactly $a(n+1)$ sets. Thus the union-closed sets conjecture would be upheld if these collections could be shown to be of boundary type.

\section{AN ORDERING ON POWER SETS}

For $k \geqslant 1$, let $\mathcal{P}(k)$ be the power set on the $k$ elements $1,2, \ldots k:$ that is, the collection of all subsets of $\{1,2, \ldots k\}$ - but excluding the null set. $P(k)$ contains $2^{k}-1$ sets, and each of the elements $1,2, \ldots k$ occurs in exactly $2^{k-1}$ sets.

Impose the following order on $\mathcal{P}(k)$. For each $A \in \mathcal{P}(k)$, assume the elements listed in increasing order. For $A, B \in \mathcal{P}(k)$ with $A=\left\{a_{1}, \ldots a_{r}\right\}, B=\left\{b_{1}, \ldots b_{s}\right\}$, $A<B$ if $a_{r}<b_{s}$; if $a_{r}=b_{\text {s }}$ then $A<B$ if $r>s$ (that is, if $A$ contains more elements); if $a_{r}=b_{\text {s }}$ and $r=s$ then $A<B$ if $A \backslash\left\{a_{r}\right\}<B \backslash\left\{b_{s}\right\}$.

Thus for example $\mathcal{P}(4)$ consists of the sets

$$
\begin{aligned}
\{1\} & <\{1,2\}<\{2\}<\{1,2,3\}<\{1,3\}<\{2,3\}<\{3\}<\{1,2,3,4\} \\
& <\{1,2,4\}<\{1,3,4\}<\{2,3,4\}<\{1,4\}<\{2,4\}<\{3,4\}<\{4\} .
\end{aligned}
$$

Note that for $k>1$ sets in $\mathcal{P}(k-1)$ precede sets in $\mathcal{P}(k) \backslash \mathcal{P}(k-1)$.

Define $\mathcal{P}(1) \backslash \mathcal{P}(0)=\{\{1\}\}$.

For positive integer $n$, choose $k$ such that $2^{k-1} \leqslant n<2^{k}$. The strictly reduced ordered power set $\mathcal{A}(n)$ is defined as the collection consisting of the first $n$ sets in $\mathcal{P}(k)$.

TheOREM 1. $\mathcal{A}(n)$ is a union-closed collection.

ProOF: If $n=2^{k}-1$ for some $k$ then $\mathcal{A}(n)=\mathcal{P}(k)$ is union-closed.

Hence assume $2^{k-1} \leqslant n<2^{k}-1$ for some $k$. Let $\mathcal{A}(n)=\mathcal{P}(k-1) \cup \mathcal{B}$. Each set in $\mathcal{B}$ contains the element $k$, and hence $\mathcal{B}$ is ordered primarily by set size, largest first. If $C \in \mathcal{P}(k-1), D \in \mathcal{B}$ then $C \cup D \in \mathcal{B}$, of size greater than or equal to $D-$ if greater, it precedes $D$; if equal it must be $D$. If $D_{1}, D_{2} \in \mathcal{B}$ then $D_{1} \cup D_{2}$ has size greater than or equal to the larger of the two sets and hence precedes it or equals it. Thus $\mathcal{A}(n)$ is union-closed.

Theorem 2 below will show that the element 1 occurs with the (possibly equal) highest frequency of all the elements in the sets in $\mathcal{A}(n)$. To aid this proof, a preliminary lemma is needed. 
LEMma 1. For $1 \leqslant r \leqslant k$ let $\mathcal{B}(r, k)$ be the collection of all sets of size $r$ in $\mathcal{P}(k) \backslash \mathcal{P}(k-1)$, ordered as above. Then removal of sets in reverse order from $\mathcal{B}(r, k)$ maintains 1 as the element with (possibly equal) highest frequency of the elements $1, \ldots k-1$ in the remaining sets in $\mathcal{B}(r, k)$.

Proof: For $r=1 \mathcal{B}(1, k)=\{\{k\}\}$ and the lemma is trivially true; for $r=2$ the sets in $\mathcal{B}(2, k)$ have order $\{1, k\}<\{2, k\}<\ldots<\{k-1, k\}$ and the lemma holds.

For $1<r \leqslant k$ the elements $1, \ldots k-1$ have equal frequency in the sets in $\mathcal{B}(r, k)$.

For $r>2$, assume for the purposes of induction that the lemma holds to $r-1$ and $k-1$. Now $\mathcal{B}(r, k)$ can be subdivided into blocks of sets of type $\{\ldots, r-1, k\}, \ldots\{\ldots, k-1, k\}$, in order as listed. For $r-1 \leqslant c \leqslant k-1$, in a block of sets of type $\{\ldots, c, k\}$ the frequency of the elements $1, \ldots c-1$ is the same.

Removal of full blocks of sets, in reverse order, from $\mathcal{B}(r, k)$ down to but not including the block $\{\ldots, c, k\}$ removes all sets containing the elements $c+1, \ldots k-1$ from $\mathcal{B}\{r, k\}$ (none if $c=k-1$ ) and reduces the frequencies of the elements $1, \ldots c$ equally, maintaining 1 as the (equally) most frequent element of $1, \ldots k-1$.

Suppose then that block $\{\ldots, c, k\}$ is at most partially reduced. At each reduction the frequency of the element $c$ is reduced by one. Initially the sets in the block contain the elements $1, \ldots c-1$ with equal frequency. The block is isomorphic to the collection $\mathcal{B}(r-1, c)$ in the sense that set $B$ occurs in the block if and only if $B \backslash\{k\} \in \mathcal{B}(r-1, c)$ and for $B_{1}, B_{2}$ sets in the block then $B_{1}<B_{2}$ if and only if $B_{1} \backslash\{k\}<B_{2} \backslash\{k\}$ in $\mathcal{B}(r-1, c)$. Thus removal of sets from this block matches removal of sets from $\mathcal{B}(r-1, c)$; but by the assumption the frequency of the element 1 diminishes most slowly of elements $1, \ldots c-1$. The lemma holds by induction.

THEOREM 2. No element occurs in sets in $\mathcal{A}(n)$ with greater frequency than the element 1 .

Proof: Select $k$ such that $2^{k-1} \leqslant n \leqslant 2^{k}-1$. Consider $\mathcal{P}(k)$.

The elements $1, \ldots k$ have equal frequency in the sets in $\mathcal{P}(k)$.

The collection $\mathcal{A}(n)$ is a reduction of $\mathcal{P}(k)$ : it can be regarded as having been created by removal from $\mathcal{P}(k)$ of sets in $\mathcal{P}(k) \backslash \mathcal{P}(k-1)$ in reverse order.

Each set removed contains the element $k$ and hence the frequency of the element $k$ diminishes fastest during this reduction.

The sets removed are ordered primarily by set size: the elements $1, \ldots k-1$ have equal frequency in each block of sets of the same size. If such a block is removed in its entirety then 1 remains the (equally) most frequent element.

Suppose a block of sets of the same size $r, 1<r<k$, is only partially removed. Then by Lemma 1 the reduction maintains 1 as the element with highest frequency in the block, of the elements $1, \ldots k-1$. 
Thus no element in $\mathcal{A}(n)$ has greater frequency than the element 1.

\section{THE FUNCTION $\psi(n)$}

For $n \geqslant 1$, define $\psi(n)$ to be the number of sets in $\mathcal{A}(n)$ which contain the element 1.

By observation on $\mathcal{P}(4)$ :

\begin{tabular}{|c|ccccccccccccccc|}
\hline$n$ & 1 & 2 & 3 & 4 & 5 & 6 & 7 & 8 & 9 & 10 & 11 & 12 & 13 & 14 & 15 \\
$\psi(n)$ & 1 & 2 & 2 & 3 & 4 & 4 & 4 & 5 & 6 & 7 & 7 & 8 & 8 & 8 & 8 \\
\hline
\end{tabular}

This leads to the conjecture that $\psi(n)=a(n+1)$; to prove this, it will be sufficient to show that for $n \geqslant 3, \psi(n)=\psi(\psi(n-1)-1)+\psi(n-\psi(n-1))$. The proof occupies most of the remainder of this paper.

\section{A partition of $\mathcal{P}(k)$}

Define the following partition of $\mathcal{P}(k), k \geqslant 1$.

For $k=1$, let $\mathcal{U}(1)$ be null, $\mathcal{V}(1)=\{\{1\}\} . \mathcal{P}(1)=\mathcal{U}(1) \cup \mathcal{V}(1)$.

For $k=2$, let $\mathcal{U}(2)=\{\{2\}\}, \mathcal{V}(2)=\{\{1,2\}\} . \mathcal{P}(2)=\mathcal{P}(1) \cup \mathcal{U}(2) \cup \mathcal{V}(2)$.

For $k \geqslant 3$, form $\mathcal{U}(k)$ and $\mathcal{V}(k)$ as follows. Adjoin the element $k$ to each set in $\mathcal{U}(2), \ldots \mathcal{U}(k-1)$; collect these sets together with the set $\{1,2, \ldots k-2, k\}(\{1,3\}$ in case $k=3$ ); apply the ordering of Section 2; call the result $\mathcal{U}(k)$. Again adjoin the element $k$ to each set in $\mathcal{V}(1), \ldots \mathcal{V}(k-1)$; collect these sets together with the set $\{k\}$; discard the set $\{1,2, \ldots k-2, k\}$; order; call the result $\mathcal{V}(k)$. Then $\mathcal{P}(k)=$ $\mathcal{P}(k-1) \cup \mathcal{U}(k) \cup \mathcal{V}(k)$.

Thus for example

$$
\begin{aligned}
& \mathcal{U}(3)=\{\{1,3\},\{2,3\}\} \\
& \mathcal{V}(3)=\{\{1,2,3\},\{3\}\} \text { (with the set }\{1,3\} \text { transferred to } \mathcal{U}(3)) \\
& \mathcal{U}(4)=\{\{1,2,4\},\{1,3,4\},\{2,3,4\},\{2,4\}\} \\
& \mathcal{V}(4)=\{\{1,2,3,4\},\{1,4\},\{3,4\},\{4\}\} \text { (with }\{1,2,4\} \text { transferred to } \mathcal{U}(4)) .
\end{aligned}
$$

This may seem a rather artificial process, but if so the fault lies in its description. Lemma 4 in the following section will show that this is in fact a natural partition, forced on $\mathcal{P}(k)$ by the definition of $\psi(n)$.

A comparison of the collections $\mathcal{U}(k), \mathcal{V}(k)$ and $\mathcal{P}(k-1) \backslash \mathcal{P}(k-2)$ is required for the proof of Theorem 3 below - this is provided in Lemmas 2 and 3 . Note for later 
FIGURE 1. Comparison of $\mathcal{P}(k-1) \backslash \mathcal{P}(k-2), \mathcal{U}(k), \mathcal{V}(k)$

\begin{tabular}{|c|c|c|c|}
\hline$k$ & $\mathcal{P}(k-1) \backslash \mathcal{P}(k-2)$ & $\overline{\mathcal{U}}(k)$ & $\overline{\mathcal{V}(k)}$ \\
\hline 1 & $-\bar{c}_{1}$ & - & $\{1\}$ \\
\hline 2 & $\{1\}$ & $\{2\}$ & $\{1,2\}$ \\
\hline \multirow[t]{2}{*}{$\overline{3}$} & $\overline{\{1,2\}}$ & 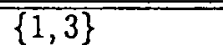 & $\{1,2,3\}$ \\
\hline & $\{2\}$ & $\{2,3\}$ & $\{3\}$ \\
\hline \multirow[t]{4}{*}{4} & $\{1,2,3\}$ & $\{1,2,4\}$ & $\{1,2,3,4\}$ \\
\hline & $\{1,3\}$ & $\{1,3,4\}$ & $\{1,4\}$ \\
\hline & $\{2,3\}$ & $\{2,3,4\}$ & $\{3,4\}$ \\
\hline & $\{3\}$ & $\{2,4\}$ & $\{4\}$ \\
\hline \multirow[t]{8}{*}{5} & $\{1,2,3,4\}$ & $\{1,2,3,5\}$ & $\{1,2,3,4,5\}$ \\
\hline & $\{1,2,4\}$ & $\{1,2,4,5\}$ & $\{1,2,5\}$ \\
\hline & $\{1,3,4\}$ & $\{1,3,4,5\}$ & $\{1,4,5\}$ \\
\hline & $\{2,3,4\}$ & $\{2,3,4,5\}$ & $\{3,4,5\}$ \\
\hline & $\{1,4\}$ & $\{1,3,5\}$ & $\{1,5\}$ \\
\hline & $\{2,4\}$ & $\{2,3,5\}$ & $\{3,5\}$ \\
\hline & $\{3,4\}$ & $\{2,4,5\}$ & $\{4,5\}$ \\
\hline & $\{4\}$ & $\{2,5\}$ & $\{5\}$ \\
\hline \multirow[t]{16}{*}{6} & $\{1,2,3,4,5\}$ & $\{1,2,3,4,6\}$ & $\{1,2,3,4,5,6\}$ \\
\hline & $\{1,2,3,5\}$ & $\{1,2,3,5,6\}$ & $\{1,2,3,6\}$ \\
\hline & $\{1,2,4,5\}$ & $\{1,2,4,5,6\}$ & $\{1,2,5,6\}$ \\
\hline & $\{1,3,4,5\}$ & $\{1,3,4,5,6\}$ & $\{1,4,5,6\}$ \\
\hline & $\{2,3,4,5\}$ & $\{2,3,4,5,6\}$ & $\{3,4,5,6\}$ \\
\hline & $\{1,2,5\}$ & $\{1,2,4,6\}$ & $\{1,2,6\}$ \\
\hline & $\{1,3,5\}$ & $\{1,3,4,6\}$ & $\{1,4,6\}$ \\
\hline & $\{2,3,5\}$ & $\{2,3,4,6\}$ & $\{3,4,6\}$ \\
\hline & $\{1,4,5\}$ & $\{1,3,5,6\}$ & $\{1,5,6\}$ \\
\hline & $\{2,4,5\}$ & $\{2,3,5,6\}$ & $\{3,5,6\}$ \\
\hline & $\{3,4,5\}$ & $\{2,4,5,6\}$ & $\{4,5,6\}$ \\
\hline & $\{1,5\}$ & $\{1,3,6\}$ & $\{1,6\}$ \\
\hline & $\{2,5\}$ & $\{2,3,6\}$ & $\{3,6\}$ \\
\hline & $\{3,5\}$ & $\{2,4,6\}$ & $\{4,6\}$ \\
\hline & $\{4,5\}$ & $\{2,5,6\}$ & $\{5,6\}$ \\
\hline & $\{5\}$ & $\{2,6\}$ & $\{6\}$ \\
\hline 7 & $\{1,2,3,4,5,6\}$ & $\{1,2,3,4,5,7\}$ & $\{1,2,3,4,5,6,7\}$ \\
\hline$\ldots$ & $\ldots \ldots$ & $\ldots \ldots$ & ...... \\
\hline
\end{tabular}

recall that for $k>3$ the first set in $\mathcal{V}(k)$ is $\{1,2, \ldots k-1, k\}$ and the last set is $\{k\}$; the first set in $\mathcal{U}(k)$ is $\{1,2, \ldots k-2, k\}$ and the last set is $\{2, k\}$. To assist understanding, refer to Figure 1.

LEMMA 2. For $k>1, \mathcal{U}(k)$ and $\mathcal{V}(k)$ contain the same number of sets. For $k>2$, when the sets in $\mathcal{U}(k)$ and $\mathcal{V}(k)$ are ordered, the element 1 is in set $i$ of $\mathcal{U}(k)$ if and only if it is also in set $i$ of $\mathcal{V}(k)$. 
PROOF: It is not difficult to show that for $k \geqslant 2$ each of $\mathcal{U}(k)$ and $\mathcal{V}(k)$ contains $2^{k-2}$ sets. However, proof of the latter part of the lemma requires close examination of the number of sets of corresponding size in each of the collections. Each of $\mathcal{U}(k)$ and $\mathcal{V}(k)$ will be subdivided into $k-1$ blocks of sets, sets within a block being of the same size, with the order in $\mathcal{U}(k)$ and $\mathcal{V}(k)$ preserved within and across the blocks. It shall then be shown that the element 1 occurs only in matching sets in these blocks.

For insight, consider the number of sets of size $s$ in $\mathcal{U}(k)$ and $\mathcal{V}(k)$ to $k=7$.

\begin{tabular}{|c|c|c|c|c|c|c|c|c|c|c|c|c|c|c|}
\hline & & & $1(k)$ & & & & & & & & (k) & & & \\
\hline$k \backslash s:$ & 1 & 2 & 3 & 4 & 5 & 6 & 7 & $k \backslash s$ & 1 & 2 & 3 & 4 & 5 & 6 \\
\hline 1 & 0 & & & & & & & 1 & 1 & & & & & \\
\hline 2 & 1 & & & & & & & 2 & 0 & 1 & & & & \\
\hline 3 & 0 & 2 & & & & & & 3 & 1 & 0 & 1 & & & \\
\hline 4 & 0 & 1 & 3 & & & & & 4 & 1 & 2 & 0 & 1 & & \\
\hline 5 & 0 & 1 & 3 & 4 & & & & 5 & 1 & 3 & 3 & 0 & 1 & \\
\hline 6 & 0 & 1 & 4 & 6 & 5 & & & 6 & 1 & 4 & 6 & 4 & 0 & 1 \\
\hline 7 & 0 & 1 & 5 & 10 & 10 & 6 & & 7 & 1 & 5 & 10 & 10 & 5 & 0 \\
\hline
\end{tabular}

Induction will first be used to prove the hypothesis that for $k \geqslant 2 \mathcal{U}(k)$ can be sudivided without loss of order into the $k-1$ blocks $\mathcal{U}(1, k), \ldots \mathcal{U}(k-1, k)$, with $\mathcal{U}(r, k)$ containing $\left(\begin{array}{c}k-2 \\ r-1\end{array}\right)$ sets, $r=1, \ldots k-1$; the set in $\mathcal{U}(1, k)$ being of size $k-1$ while for $r>1$ the sets in $\mathcal{U}(r, k)$ are of size $k-r+1$.

This holds at $k=2$ : the single block $\mathcal{U}(1,2)$ then contains the set $\{2\}$ of size 1 .

For $k=3, \mathcal{U}(3)$ can be split into $\mathcal{U}(1,3)$ and $\mathcal{U}(2,3)$ with $\mathcal{U}(1,3)$ containing the set $\{1,3\}$, preceding the set $\{2,3\}$ in $U(2,3)$.

For $k=4$, split $\mathcal{U}(4)$ into

$$
\begin{aligned}
& \mathcal{U}(1,4)=\{\{1,2,4\}\} \\
& \mathcal{U}(2,4)=\{\{1,3,4\},\{2,3,4\}\} \\
& \mathcal{U}(3,4)=\{\{2,4\}\}
\end{aligned}
$$

For $k>4$ : assume the hypothesis valid to $k-1$. Now by definition $\mathcal{U}(k)$ is constructed from $\mathcal{U}(2)$ to $\mathcal{U}(k-1)$ by adjoining the element $k$ to each set in these collections, and adding the set $\{1,2, \ldots k-2, k\}$. This set, of size $k-1$, precedes all other sets in $\mathcal{U}(k)$ : place it in block $\mathcal{U}(1, k)$.

$\mathcal{U}(k)$ has no sets of size one. By construction, it has exactly one set of size two, $\{2, k\}$ derived from $\{2\}$ in $\mathcal{U}(2)$ : place it in block $\mathcal{U}(k-1, k)$. 
The remainder of $\mathcal{U}(k)$ consists of sets constructed from those in $\mathcal{U}(3)$ to $\mathcal{U}(k-1)$ $\rightarrow$ size 2 to $k-2$ by the induction assumption, leading to sets of size 3 to $k-1$ in $\mathcal{U}(k)$.

Place in $\mathcal{U}(r, k)$ sets of size $k-r+1, r=2, \ldots k-2$. These are constructed from sets of size $k-r$, which by the assumption occur in $\mathcal{U}(1, k-r+1)$ and $\mathcal{U}(2, k-r+1)$, also in $\mathcal{U}(3, k-r+2), \ldots \mathcal{U}(r, k-1)$.

Thus for $2 \leqslant r \leqslant k-2$ the block $\mathcal{U}(r, k)$ contains

$$
1+\left(\begin{array}{c}
k-r-1 \\
1
\end{array}\right)+\left(\begin{array}{c}
k-r \\
2
\end{array}\right)+\cdots+\left(\begin{array}{c}
k-3 \\
r-1
\end{array}\right)=\sum_{i=0}^{r-1}\left(\begin{array}{c}
k-r-2+i \\
i
\end{array}\right)=\left(\begin{array}{c}
k-2 \\
r-1
\end{array}\right) \text { sets. }
$$

Previously the blocks $\mathcal{U}(1, k)$ and $\mathcal{U}(k-1, k)$ were constructed, holding $\{1,2, \ldots k-2, k\}$ and $\{2, k\}$ respectively. Since primary ordering in $\mathcal{U}(k)$ is by length, the induction hypothesis is now validated.

This result also shows that $\mathcal{U}(k)$ contains $2^{k-2}$ sets, $k \geqslant 2$.

A similar inductive process can now be used to prove the hypothesis that for $k \geqslant 2$ $\mathcal{V}(k)$ can be subdivided into the $k-1$ blocks $\mathcal{V}(1, k), \ldots \mathcal{V}(k-1, k)$ with one set of size $k$ in $\mathcal{V}(1, k)$ and $\left(\begin{array}{c}k-2 \\ r-1\end{array}\right)$ sets of size $k-r$ in $\mathcal{V}(r, k)$ for $r=2, \ldots k-1$.

This is valid at $k=2: \mathcal{V}(2)$ has a single block $\mathcal{V}(1,2)$ containing the set $\{1,2\}$.

At $k=3, \mathcal{V}(3)$ has blocks $\mathcal{V}(1,3)$ and $\mathcal{V}(2,3)$ containing $\{1,2,3\}$ and $\{3\}$ respectively as required.

For $k \geqslant 4$, assume the hypothesis valid to $k-1$. Recall $\mathcal{V}(k)$ is constructed from $\mathcal{V}(1)$ to $\mathcal{V}(k-1)$ by adjoining the element $k$ to each set, discarding the set $\{1,2, \ldots k-2, k\}$, and adding the set $\{k\}$. It contains one set of size $k,\{1,2, \ldots k\}$ : insert this into block $\mathcal{V}(1, k) . \mathcal{V}(k)$ contains also one set of size $1,\{k\}$ : insert this into $\mathcal{V}(k-1, k)$.

The remaining sets are of size 2 to $k-2$, constructed from sets in $\mathcal{V}(1)$ to $\mathcal{V}(k-1)$ of size 1 to $k-3$ (the set $\{1,2, \ldots k-2, k\}$ of size $k-1$ arising from $\mathcal{V}(k-2)$ being discarded). These can be inserted into blocks $\mathcal{V}(2, k)$ to $\mathcal{V}(k-2, k)$, sets in $\mathcal{V}(r, k)$ having size $k-r$.

By the inductive assumption, for $2 \leqslant r \leqslant k-2$, sets of size $k-r-1$ occur in $\mathcal{V}(1, k-r-1)$ and $\mathcal{V}(2, k-r+1), \mathcal{V}(3, k-r+2), \ldots \mathcal{V}(r, k-1)$. These form sets of size $k-r$ in $\mathcal{V}(k)$ : thus the block $\mathcal{V}(r, k)$ also contains

$$
1+\left(\begin{array}{c}
k-r-1 \\
1
\end{array}\right)+\left(\begin{array}{c}
k-r \\
2
\end{array}\right)+\cdots+\left(\begin{array}{l}
k-3 \\
r-1
\end{array}\right)=\left(\begin{array}{c}
k-2 \\
r-1
\end{array}\right) \text { sets. }
$$

Recall $\mathcal{V}(1, k)$ and $\mathcal{V}(k-1, k)$ were constructed separately, each containing one set. This result thus also shows that $\mathcal{V}(k)$ contains $2^{k-2}$ sets. 
Thus $\mathcal{U}(k)$ and $\mathcal{V}(k)$ each split into $k-1$ ordered blocks, matching blocks containing the same number of sets. The order of the blocks is by set size (sets in $\mathcal{U}(1, k)$ and $\mathcal{U}(2, k)$ having the same size); order within the blocks is that within $\mathcal{U}(k)$ and $\mathcal{V}(k)$.

Turn now to the correspondence of sets containing the element 1 . To complete the proof of this lemma it will now be sufficient to prove the hypothesis that, for $k>2$, sets containing the element 1 match up in corresponding blocks.

For $k=3$, the blocks in $\mathcal{U}(3)$ are $\mathcal{U}(1,3)=\{\{1,3\}\}$ and $\mathcal{U}(2,3)=\{\{2,3\}\}$ while those in $\mathcal{V}(3)$ are $\mathcal{V}(1,3)=\{\{1,2,3\}\}$ and $\mathcal{V}(2,3)=\{\{3\}\}$, with correspondence for the element 1 .

Consider $\mathcal{U}(k)$ and $\mathcal{V}(k)$ for $k>3$.

Assume for inductive purposes that matching blocks in $\mathcal{U}(3)$ and $\mathcal{V}(3)$ to $\mathcal{U}(k-1)$ and $\mathcal{V}(k-1)$ satisfy the corresponding property for element 1 .

$\mathcal{U}(1, k)$ contains the set $\{1,2, \ldots k-2, k\}$, matching the set $\{1,2, \ldots k\}$ in $\mathcal{V}(1, k)$.

$\mathcal{U}(k-1, k)$ contains $\{2, k\}$ matching $\{k\}$ in $\mathcal{V}(k-1, k)$.

Thus consider $\mathcal{U}(r, k)$ and $\mathcal{V}(r, k)$ for $2 \leqslant r \leqslant k-2$.

As previously noted, $\mathcal{U}(r, k)$ is constructed from $\mathcal{U}(1, k-r+1) ; \mathcal{U}(2, k-r+1)$, $\mathcal{U}(3, k-r+2), \ldots \mathcal{U}(r, k-1)$ while $\mathcal{V}(r, k)$ is constructed from $\mathcal{V}(1, k-r-1)$; $\mathcal{V}(2, k-r+1), \mathcal{V}(3, k-r+2), \ldots \mathcal{V}(r, k-1)$. The number of sets in corresponding blocks is the same. Since all sets in $\mathcal{U}(r, k)$ have the same size and contain the element $k$, their order is primarily by the second-last element in each set, or by block of origin, secondarily by order within that block; similarly for $\mathcal{V}(r, k)$.

The induction assumption has sets containing the element 1 matching across all these blocks, except possibly the first.

But the set in $\mathcal{U}(1, k-r+1)$ is $\{1,2, \ldots k-r-1, k-r+1\}$, matching the set $\{1,2, \ldots k-r-1\}$ in $\mathcal{V}(1, k-r-1)$.

Thus the second part of the lemma holds by induction.

Lemma 3. For $k \geqslant 2, \mathcal{V}(k)$ and $\mathcal{P}(k-1) \backslash \mathcal{P}(k-2)$ contain the same number of sets. When the collections are ordered, the element 1 is in set $i$ of $\mathcal{V}(k)$ if and only if it is also in set $i$ of $\mathcal{P}(k-1) \backslash \mathcal{P}(k-2)$.

Proof: It is not difficult to show that for $k \geqslant 2, \mathcal{P}(k-1) \backslash \mathcal{P}(k-2)$ contains $2^{k-2}$ sets, as does $\mathcal{V}(k)$ by Lemma 2. However, the matching of sets containing the element 1 again requires deeper analysis, here of the block structure in $\mathcal{P}(k-1) \backslash \mathcal{P}(k-2)$ and $\mathcal{V}(k)$, matching blocks and sets within these blocks.

By definition $\mathcal{P}(1) \backslash \mathcal{P}(0)=\{\{1\}\} ;$ this matches with $\mathcal{V}(2)=\{\{1,2\}\}$.

The sets in $\mathcal{P}(2) \backslash \mathcal{P}(1)$ have order $\{1,2\}<\{2\}$, while those in $\mathcal{V}(3)$ have order $\{1,2,3\}<\{3\}$. 
For $k>2$ the inner structure of $\mathcal{P}(k-1) \backslash \mathcal{P}(k-2)$ is isomorphic to that of $\mathcal{P}(k-2) \cup\{0\}$ in that $C \in \mathcal{P}(k-1) \backslash \mathcal{P}(k-2)$ if and only if $C \backslash\{k-1\} \in \mathcal{P}(k-2) \cup\{0\}$. Subdivided by set size $\mathcal{P}(k-1) \backslash \mathcal{P}(k-2)$ consists of $k-1$ blocks. In decreasing order label these $\mathcal{C}(1, k-1)$ to $\mathcal{C}(k-1, k-1)$, where $\mathcal{C}(r, k-1)$ contains the $\left(\begin{array}{l}k-2 \\ r-1\end{array}\right)$ sets of size $k-r, r=1, \ldots k-1$. The number of sets in $\mathcal{P}(k-1) \backslash \mathcal{P}(k-2)$ is $2^{k-2}$.

Recall from Lemma 2 that $\mathcal{V}(k)$ has block structure consisting of $\mathcal{V}(1, k)$ with one set of size $k$, followed for $r=2, \ldots k-1$ by blocks $\mathcal{V}(r, k)$ containing $\left(\begin{array}{c}k-2 \\ r-1\end{array}\right)$ sets of size $k-r$.

The block structure of these two collections thus matches, with corresponding blocks $\mathcal{C}(r, k-1)$ and $\mathcal{V}(r, k)$ containing the same number of sets. For example, below is shown the number of sets of size $s$ in the first few collections.

$\mathcal{V}(k) \quad \mathcal{P}(k-1) \backslash \mathcal{P}(k-2)$

$\begin{array}{lrlrrrrrrrrrrrrr}k \backslash s: & 1 & 2 & 3 & 4 & 5 & 6 & 7 & k \backslash s: & 1 & 2 & 3 & 4 & 5 & 6 & 7 \\ 1 & 1 & & & & & & & 1 & & & & & & & \\ 2 & 0 & 1 & & & & & & 2 & 1 & & & & & & \\ 3 & 1 & 0 & 1 & & & & & 3 & 1 & 1 & & & & & \\ 4 & 1 & 2 & 0 & 1 & & & & 4 & 1 & 2 & 1 & & & \\ 5 & 1 & 3 & 3 & 0 & 1 & & & 5 & 1 & 3 & 3 & 1 & & \\ 6 & 1 & 4 & 6 & 4 & 0 & 1 & & 6 & 1 & 4 & 6 & 4 & 1 & & \\ 7 & 1 & 5 & 10 & 10 & 5 & 0 & 1 & 7 & 1 & 5 & 10 & 10 & 5 & 1 & \end{array}$

For the purpose of this proof, for $k>2 \mathcal{P}(k-1) \backslash \mathcal{P}(k-2)$ can be regarded as having been formed by adjoining the element $k-1$ to each set in $\mathcal{P}(1) \backslash \mathcal{P}(0)$ to $\mathcal{P}(k-2) \backslash \mathcal{P}(k-3)$; adding the set $\{k-1\}$; then ordering.

Recall $\mathcal{V}(k)$ is constructed by adjoining the element $k$ to each set in $\mathcal{V}(1)$ to $\mathcal{V}(k-1)$; discarding the set $\{1,2, \ldots k-2, k\}$; adding the set $\{k\}$; then ordering.

Since the number of sets in corresponding blocks in $\mathcal{P}(k-1) \backslash \mathcal{P}(k-2)$ and $\mathcal{V}(k)$ matches, it will now be sufficient to prove the hypothesis that corresponding blocks have the matching property for element 1 . This is true at $k=2$ and $k=3$; for the purpose of induction assume it true to $k-1$ for some $k>3$.

$\mathcal{C}(1, k-1)$ contains the set $\{1,2, \ldots k-1\}$, matching $\{1,2, \ldots k\}$ in $\mathcal{V}(1, k)$. $\mathcal{C}(k-1, k-1)$ contains the set $\{k-1\}$, matching $\{k\}$ in $\mathcal{V}(k-1, k)$.

For $2 \leqslant r \leqslant k-2$, the sets in block $\mathcal{C}(r, k-1)$ can be constructed from sets in $\mathcal{C}(1, k-r-1), \mathcal{C}(2, k-r), \ldots \mathcal{C}(r, k-2)$ while those in $\mathcal{V}(r, k)$ are constructed from sets in $\mathcal{V}(1, k-r-1) ; \mathcal{V}(2, k-r+1), \ldots \mathcal{V}(r, k-1)$. The number of sets constructed at each step matches. Since all sets in $\mathcal{C}(r, k-1)$ have the same size and the same last element, their order is primarily by second-last element and hence by block of origin, secondarily by order within that block; similarly for $\mathcal{V}(r, k)$. 
The induction assumption matches sets containing the element 1 across all blocks containing these originating sets, except for the first.

But $\mathcal{C}(1, k-r-1)$ contains the set $\{1,2, \ldots k-r-1\}$, identical to the set in $\mathcal{V}(1, k-r-1)$.

Thus the lemma follows by induction - sets containing the element 1 match by position in $\mathcal{P}(k-1) \backslash \mathcal{P}(k-2)$ and $\mathcal{V}(k)$.

\section{The recurRence relation on $\psi(n)$}

The following lemma explains the partitioning of $\mathcal{P}(k) \backslash \mathcal{P}(k-1)$ into $\mathcal{U}(k)$ and $\mathcal{V}(k)$.

Lemma 4. Let $A_{n}$ be the $n$th set in an ordered power set. For $n>2$, if $A_{n} \in$ $\mathcal{U}(k)$ for some $k$ then $\psi(n-1)=\psi(n-2)+1$ while if $A_{n} \in \mathcal{V}(k)$ then $\psi(n-1)=$ $\psi(n-2)$.

Proof: Consider first the case $A_{n} \in \mathcal{U}(k)$. The starting level for $\mathcal{U}$-sets is $\{2\}$ in $\mathcal{U}(2)$, which has predecessor $\{1,2\}$ in $\mathcal{A}_{n}$; this leads to $\{2,3\}$ in $\mathcal{U}(3)$, with $\{1,3\}$ added: predecessors $\{1,3\}$ and $\{1,2,3\}$ respectively. For $k>3$ sets in $\mathcal{U}(k)$ are formed by adjoining $k$ to all sets at lower levels, and adding the set $\{1,2, \ldots k-2, k\}$ - thus each set in $\mathcal{U}(k)$ has one of the following forms:

(i) $\{2, k\}:$ predecessor $\{1, k\}$

(ii) $\{2, c, \ldots k\}$ : predecessor $\{1, c, \ldots k\}$

(iii) $\{1,3, k\}$ : predecessor $\{1,2, k\}$

(iv) $\{1,3, c, \ldots k\}$ predecessor $\{1,2, c, \ldots k\}$

(v) $\{1,2, \ldots i-2, i, c, \ldots k\}$ : predecessor $\{1,2, \ldots i-2, i-1, c, \ldots k\}$

(vi) $\{1,2, \ldots k-2, k\}$ : predecessor $\{1,2, \ldots k-2, k-1, k\}$.

Thus if $A_{n} \in \mathcal{U}(k)$ then $1 \in A_{n-1}$, and $\psi(n-1)=\psi(n-2)+1$.

Consider now $A_{n} \in \mathcal{V}(k), n>2$. For $k=1$ the starting level is $\mathcal{V}(1)$ containing $A_{1}=\{1\}$; for $k=2$ the single set in $\mathcal{V}(2)$ is $A_{2}=\{1,2\}$. For $k=3$ there are two sets in $\mathcal{V}(k),\{3\}$ and $\{1,2,3\}$ with predecessors $\{2,3\}$ and $\{2\}$ respectively. For $k>3$ sets in $\mathcal{V}(k)$ are formed by adjoining $k$ to sets from lower levels, discarding the set $\{1,2, \ldots k-2, k\}$, adding the set $\{k\}$. Thus each set in $\mathcal{V}(k)$ has one of the following forms:

(i) $\{1, k\}$ : predecessor $\{k-2, k-1, k\}$ with $k-2>1$

(ii) $\left\{1, c_{1}, c_{2}, \ldots k\right\}, c_{1}>3$ : predecessor $\left\{c_{1}-2, c_{1}-1, c_{2}, \ldots k\right\}$

(iii) $\{1,2, \ldots i, k\}$, with the first $i$ natural numbers and $k>i+2$ : predecessor $\{k-i-1, k-i, \ldots k-1, k\}$

(iv) $\{1,2, \ldots i, c, \ldots k\}$, with the first $i$ natural numbers and $c>i+2$ : predecessor $\{c-i-1, c-i, \ldots c-2, c-1, \ldots k\}$ 
(v) $\left\{c_{1}, c_{2}, \ldots k\right\}, c_{1} \geqslant 3$ : predecessor $\left\{c_{1}-1, c_{2}, \ldots k\right\}$

(vi) $\{1,2, \ldots k-1, k\}$ : predecessor $\{k-1\}$.

Thus for $n>2$ if $A_{n} \in \mathcal{V}\{k\}$ then $1 \notin A_{n-1}$ and $\psi(n-1)=\psi(n-2)$.

We are now in a position to prove the main theorem.

ThEOREM 3. For $n>2, \psi(n)=\psi(\psi(n-1)-1)+\psi(n-\psi(n-1))$.

Proof: Let $\mathcal{A}(n)=\left\{A_{1}, A_{2}, \ldots A_{n}\right\}$ be a strictly reduced ordered power set. Select $k$ such that $2^{k-1} \leqslant n \leqslant 2^{k}-1 . \psi(n)$ equals the number of sets in $\mathcal{A}(n)$ which contain the element 1 : but $\mathcal{A}(n)$ can be partitioned into sets in $\mathcal{U}(i)$ and $\mathcal{V}(i)$, $i=1, \ldots k$ and thus $\psi(n)$ is the sum of the number of $\mathcal{U}$-sets and $\mathcal{V}$-sets in $\mathcal{A}(n)$ which contain a 1 . By Lemma 4 each $\mathcal{U}$-set in $\mathcal{A}(n)$ is preceded by a set containing a 1 , while no $\mathcal{V}$-set apart from the set $\{1,2\}$ is preceded by a set containing a 1 . The number of $\mathcal{U}$-sets in $\mathcal{A}(n)$ is thus equal to the number of sets containing a 1 in $\mathcal{A}(n-1)$, minus one for the set $\{1,2\}$ : thus the number of $\mathcal{U}$-sets in $\mathcal{A}(n)$ is $\psi(n-1)-1$. The number of $\mathcal{V}$-sets in $\mathcal{A}(n)$ is now $n+1-\psi(n-1)$.

$\psi(n)$ is equal to the sum of the number of $\mathcal{U}$-sets and $\mathcal{V}$-sets in $\mathcal{A}(n)$ containing a 1 . By Lemma 3, the positioning of sets containing the element 1 in $\mathcal{V}(i)$ is that in $\mathcal{P}(i-1) \backslash \mathcal{P}(i-2), i \geqslant 2$ : by Lemma 2 this is also the positioning $\mathcal{U}(i)$ for $i \geqslant 3$.

The number of the $\psi(n-1)-1 \mathcal{U}$-sets containing the element 1 is thus the same as the number in $\mathcal{A}(\psi(n-1)-1)$, minus one for the non-correspondence of $\mathcal{U}(2)=\{\{2\}\}$ with $\mathcal{P}(1) \backslash \mathcal{P}(0)=\{\{1\}\}: \psi(\psi(n-1)-1)-1 \mathcal{U}$-sets contain the element 1 .

The number of $\mathcal{V}$-sets containing the element 1 is made up of one from $\mathcal{V}(1)=$ $\{\{1\}\}$, then the number in the remaining $n-\psi(n-1) \mathcal{V}$-sets, which equals the number in $\mathcal{A}(n-\psi(n-1))$ by Lemma 3. Thus $1+\psi(n-\psi(n-1)) \mathcal{V}$-sets contain the element 1 .

Thus $\psi(n)=\psi(\psi(n-1)-1)+\psi(n-\psi(n-1))$.

\section{Discussion}

We have created union-closed sets $\mathcal{A}(n)$ of a certain type, in which the element with highest frequency occurs in exactly $\psi(n)=a(n+1)$ sets. This leads immediately to the result $\phi(n) \leqslant \psi(n)$ where $\phi(n)$ is Renaud's boundary function, and to the conjecture $\phi(n)=\psi(n)$. To prove equality it would be necesary to show that the strictly reduced ordered power sets defined here are of boundary type, but while this seems reasonable it is not immediate. There exist union-closed collections of boundary type which are not lattice isomorphic to an appropriate $\mathcal{A}(n)$ - for example, the collection $\mathcal{P}(4)$ with all singletons removed is not lattice isomorphic to $\mathcal{A}(11)$, although it has maximum frequency 7 ; this can be seen by noticing that no reduction on this collection by two sets leads to a union-closed collection of nine sets of boundary type. A possible line of 
attack would be to express any boundary union-closed collection as a subset of some $\mathcal{A}(m)$ and attempt transformations on it which do not affect the maximum frequency, leading finally to some $\mathcal{A}(n)$, but it is not known if this is feasible.

\section{REFERENCES}

[1] C.I. Mallows, 'Conway's challenge sequence', Amer. Math. Monthly 98 (1991), 5-20.

[2] J-C. Renaud, 'Is the union-closed sets conjecture the best possible?', J. Austral. Math. Soc. Ser. A 51 (1991), 276-283.

[3] I. Rival (Editor), Graphs and order (Reidel, Dordrecht, 1984).

Department of Mathematics

University of Papua New Guinea

PO Box 320

University

Papua New Guinea 